Journal of Mathematics and Informatics

Vol. 11, 2017, 29-37

ISSN: 2349-0632 (P), 2349-0640 (online)

Published 11 December 2017

www.researchmathsci.org

DOI: http://dx.doi.org/10.22457/jmi.v1la5

Journal of

Mathematics and

Informatics

\title{
On The Homogeneous Cubic Equation With Four
} Unknowns $\left(x^{3}+y^{3}\right)=7 z w^{2}$

\section{C.Pathmapriya $^{1}$ and G.Sumathi ${ }^{2}$}

Department of Mathematics, Shrimati Indira Gandhi College

Trichy-2, Tamilnadu, India. 'e-mail: cspathmapriya025@ gmail.com

e-mail: b.deepacharan@gmaill.com

Received 1 November 2017; accepted 8 December 2017

Abstract. The homogeneous cubic equation with four unknowns represented by the Diophantine equation $\left(x^{3}+y^{3}\right)=7 z w^{2}$ is analyzed for its patterns of non-zero distinct integer solutions. A few interesting properties between the solutions and special numbers are exhibited.

Keywords: Homogeneous cubic, cubic with four unknowns, integral solutions.

AMS Mathematics Subject Classification (2010): 11D25

\section{Introduction}

The Diophantine equation offer an unlimited field for research due to their variety [1-2]. In particular, one may refer [3-7] for the cubic equation with three and four unknowns. This communication concerns with yet another interesting equation $\left(x^{3}+y^{3}\right)=7 z w^{2}$ representing homogeneous cubic with four unknowns for determining its infinitely many non-zero integral points, also a few interesting relations among the solutions are presented.

\section{Notations}

1) Polygonal number of rank ' $n$ ' with m sides

$$
t_{m, n}=n\left(1+\left(\frac{(n-1)(m-2)}{2}\right)\right)
$$

\section{2) Jacobsthal-Lucas number of rank $n$}

$$
j_{n}=2^{n}+(-1)^{n}
$$

3) Pronic number of rank ' $n$ '

$$
P R_{n}=n(n-1)
$$

4) Centered Polygonal number of rank ' $n$ ' with $m$ sides 


$$
C t_{m, n}=\frac{m n(n-1)+2}{2}
$$

\section{5) Centered hexagonal Pyramidal number of rank ' $n$ '}

$$
C P_{n, 6}=n^{3}
$$

\section{Method of analysis}

The equation representing the homogeneous cubic equation to be solved for its non-zero distinct integer solution

$\left(x^{3}+y^{3}\right)=7 z w^{2}$

It is to be noted that, (1) is satisfied by the following two integer quadruples

$(76 k, 4 k, 80 k, 28 k),(32 k,-16 k, 16 k, 16 k)$

The substitution of linear transformation

$x=u+v, y=u-v, z=2 u, u \neq v \neq 0$

in (1) leads to

$u^{2}+3 v^{2}=7 w^{2}$

Assume that $w=a^{2}+3 b^{2}$, where $a, b \succ 0$

\subsection{Pattern-1}

Write7 as,

$$
7=(2+i \sqrt{3})(2-i \sqrt{3})
$$

Substituting (4) and (5) in (3)

Using the method of factorization, we get

$(u+i \sqrt{3} v)(u-i \sqrt{3} v)=(a+i \sqrt{3} b)^{2}(a-i \sqrt{3} b)^{2}(2+i \sqrt{3})(2-i \sqrt{3})$

Equating the positive and negative factors, the resulting equations are

$(u+i \sqrt{3} v)=(2+i \sqrt{3})(a+i \sqrt{3} b)^{2}$

$(u-i \sqrt{3} v)=(2-i \sqrt{3})(a-i \sqrt{3} b)^{2}$

Equating the real and imaginary parts, we have

$$
\left.\begin{array}{c}
u=2 a^{2}-6 b^{2}-6 a b \\
v=a^{2}-3 b^{2}+4 a b
\end{array}\right\}
$$

Hence in view of (2), the non-zero distinct integer values of $x, y, z, w$ of (1) are given by

$$
\begin{aligned}
& x=3 a^{2}-9 b^{2}-2 a b \\
& y=a^{2}-3 b^{2}-10 a b \\
& z=4 a^{2}-12 a b-12 b^{2} \\
& w=a^{2}+3 b^{2}
\end{aligned}
$$

\section{Properties:}

1.x $x(a, a)-3 y(a, a)-28 t_{4, a}=0$

2. $y(a, a+1)+w(a, a+1)-2 t_{4, a}+20 t_{3, a}=0$

$3.6[w(1,1)]$ is a nasty number 


\section{On the homogeneous cubic equation with four unknowns}

$$
\left(x^{3}+y^{3}\right)=7 z w^{2}
$$

\subsection{Pattern-2}

Instead of (5), 7 can be written as

$7=\frac{(5+i \sqrt{3})(5-i \sqrt{3})}{4}$

Proceeding as in pattern: 1, the non-zero distinct integer solution to (1) are given by

$x=3 a^{2}-9 b^{2}+2 a b$

$y=2 a^{2}-6 b^{2}-8 a b$

$z=5 a^{2}-15 b^{2}-6 a b$

$w=a^{2}+3 b^{2}$

\section{Properties:}

1.w $(a, a)-,4 t_{4, a}=0$

$2 . x(a, 1)+y(a, 1)-t_{12, a} \equiv 1(\bmod 8)$

$3 . z(1,1)+j_{4}-1=0$

\subsection{Pattern-3}

The substitution of linear transformation

$w=X+3 T, V=X+7 T, u=2 U$

in (3) leads to

$U^{2}=X^{2}-21 T^{2}$

$X^{2}-U^{2}=21 T^{2}$

write (11) as

$(X+U)(X-U)=21 T^{2}$

The equation (12) is written as the system of two equations as follows:

\begin{tabular}{|l|l|l|l|}
\hline System & $\mathbf{1}$ & $\mathbf{2}$ & $\mathbf{3}$ \\
\hline$X+U$ & 21 & $3 T^{2}$ & $7 T^{2}$ \\
\hline$X-U$ & $T^{2}$ & 7 & 3 \\
\hline
\end{tabular}

\section{System 1:}

Consider

$X+U=21$

$X-U=T^{2}$

Solving these two equations we get

$$
\left.\begin{array}{l}
X=2 k^{2}+12 k+38 \\
U=-2 k^{2}-2 k+20 \\
T=2 k+1
\end{array}\right\}
$$

(13)Substituting (13) in (10) and (2), we get the corresponding non-zero distinct integer solutions to (1) as follows:

$x=-2 k^{2}+12 k+38$

$y=-6 k^{2}-20 k+2$ 


\section{C.Pathmapriya and Dr.G.Sumathi}

$z=40-8 k^{2}-8 k$

$w=2 k^{2}+8 k+14$

\section{Properties:}

$$
\begin{aligned}
& 1 . x(k)-y(k)-t_{10, k} \equiv 1(\bmod 35) \\
& 2 . x(k)+y(k)+16 t_{3, k} \equiv 0(\bmod 5) \\
& 3 . z+2 w+4 t_{4, k} \equiv 4(\bmod 8)
\end{aligned}
$$

\section{System 2:}

\section{Consider,}

$X+U=3 T^{2}$

$X-U=7$

Solving these two equations we get

$$
\left.\begin{array}{l}
X=6 k^{2}-6 k+5 \\
U=6 k^{2}-6 k-2 \\
T=2 k-1
\end{array}\right\}
$$

Substituting equation (14) in (10) and (2), we get the corresponding non-zero distinct integer solutions to (1) as follows

$$
\begin{aligned}
& x=18 k^{2}-4 k-6 \\
& y=6 k^{2}-20 k-2 \\
& z=24 k^{2}-24 k-8 \\
& w=6 k^{2}+2
\end{aligned}
$$

\section{Properties:}

1.x(1) $-C P_{2,6}=6$

$2 . x(k)+w(k)-t_{50, k} \equiv-4(\bmod 19)$

$3.6[2 w(1)]$ is a nasty number

\section{System 3:}

\section{Consider,}

$$
\begin{aligned}
& X+U=7 T^{2} \\
& X-U=3
\end{aligned}
$$

Solving these two equations we get

$$
\left.\begin{array}{l}
X=14 k^{2}+14 k+5 \\
U=14 k^{2}+14 k+2 \\
T=2 k+1
\end{array}\right\}
$$

Substituting (15) in (10) and (2), we get the corresponding non-zero distinct integer solutions to (1) as follows

$$
\begin{aligned}
& x=42 k^{2}+56 k+16 \\
& y=14 k^{2}-8 \\
& z=56 k^{2}+56 k+8 \\
& w=14 k^{2}+20 k+8
\end{aligned}
$$




\section{On the homogeneous cubic equation with four unknowns}

$$
\left(x^{3}+y^{3}\right)=7 z w^{2}
$$

\section{Properties:}

$1.6[y(1)-2]$ is a nasty number

$2 . x(k)+y(k)-56 P R_{k}-8=0$

$3 . y(k)+w(k)-28 P R_{k} \equiv 0(\bmod 8)$

\subsection{Pattern-4}

(11) can be re-written as

$X^{2}=21 T^{2}+U^{2}$

which is satisfied by

$$
\left.\begin{array}{l}
X=21 m^{2}+n^{2} \\
U=21 m^{2}-n^{2} \\
T=2 m n
\end{array}\right\}
$$

Using (16) in (11) we get

$u=42 m^{2}-2 n^{2}$

$v=21 m^{2}+14 m n+n^{2}$

$w=21 m^{2}+6 m n+n^{2}$

Thus in view (2) and (3), the non-zero distinct integer solutions to (1) are obtained by

$$
\begin{aligned}
& x=63 m^{2}-n^{2}+14 m n \\
& y=21 m^{2}-3 n^{2}+14 m n \\
& z=84 m^{2}-4 n^{2} \\
& w=21 m^{2}+n^{2}+6 m n
\end{aligned}
$$

\section{Properties:}

$$
\begin{aligned}
& 1 . y(1, n)-w(1, n)+4 t_{4, n} \equiv 0(\bmod 8) \\
& 2 . w(m, 1)-t_{44, m}-1 \equiv 0(\bmod 26) \\
& \text { 3.w }(m, m+1)-t_{154, m}+1 \equiv 0(\bmod 87)
\end{aligned}
$$

\subsection{Pattern-5}

Consider (12) as

$$
(X+U)(X-U)=21 T^{2}
$$

\section{Case: 1}

Write (18) in the form of ratio as $\frac{(X+U)}{T}=\frac{21 T}{(X-U)}=\frac{\alpha}{\beta}, \beta \neq 0$

which is equivalent to the following two equations

$$
\begin{aligned}
& \beta X+\beta U-\alpha T=0 \\
& \alpha X-\alpha U-21 \beta T=0
\end{aligned}
$$


C.Pathmapriya and Dr.G.Sumathi

On employing the method of cross multiplication, we get

$U=\alpha^{2}-21 \beta^{2}$

$X=21 \beta^{2}+\alpha^{2}$

$T=2 \alpha \beta$

Thus, in view of (10) we get

$u=2 \alpha^{2}-42 \beta^{2}$

$v=\alpha^{2}+14 \alpha \beta+21 \beta^{2}$

$w=\alpha^{2}+6 \alpha \beta+21 \beta^{2}$

Substituting in (2), we get

$\left.\begin{array}{l}x=x(\alpha, \beta)=3 \alpha^{2}+14 \alpha \beta-21 \beta^{2} \\ y=y(\alpha, \beta)=\alpha^{2}-14 \alpha \beta-63 \beta^{2} \\ z=z(\alpha, \beta)=4 \alpha^{2}-84 \beta^{2} \\ w=w(\alpha, \beta)=\alpha^{2}+6 \alpha \beta+21 \beta^{2}\end{array}\right\}$

Thus (20) represents non-zero distinct integer solution of (1)

\section{Properties:}

1. $x(\alpha, 1)+y(\alpha, 1)-2 t_{4, \alpha} \equiv 0(\bmod 2)$

$2 . z(\beta+1, \beta)+80 t_{4, \alpha} \equiv 4(\bmod 8)$

3. $z(\alpha, 1)-y(\alpha, 1)-t_{8, \alpha} \equiv 11(\bmod 16)$

Case 2:

Write (18) in the form of ratio as, $\frac{(X+U)}{3 T}=\frac{7 T}{(X-U)}=\frac{\alpha}{\beta}, \beta \neq 0$

Proceeding as in case (1), we get

$x=x(\alpha, \beta)=9 \alpha^{2}+14 \alpha \beta-7 \beta^{2}$

$y=y(\alpha, \beta)=3 \alpha^{2}-14 \alpha \beta-21 \beta^{2}$

$z=z(\alpha, \beta)=12 \alpha^{2}-28 \beta^{2}$

$w=w(\alpha, \beta)=3 \alpha^{2}+6 \alpha \beta+7 \beta^{2}$

Thus (21) represents the non-zero distinct integer solution of (1)

\section{Properties:}

1. $x(\alpha, 1)-y(\alpha, 1)-t_{14, \alpha} \equiv 14(\bmod 33)$

$2 . z(\alpha, 1)-12 P R_{\alpha} \equiv 8(\bmod 12)$

3. $x(\alpha, \alpha)+y(\alpha, \alpha)+16 t_{4, \alpha}=0$

\section{Remarkable observations}

Triple 1:

Let $u_{0}, v_{0}, w_{0}$ be the initial solution of (3) 


\section{On the homogeneous cubic equation with four unknowns}

$$
\left(x^{3}+y^{3}\right)=7 z w^{2}
$$

$\left.\begin{array}{l}u_{1}=u_{0} \\ v_{1}=v_{0}+3 h \\ w_{1}=w_{0}+2 h\end{array}\right\}$

be the second solution of (3), where $h$ is a non-zero integer to be determined.

Then from(3), we get

$$
\begin{aligned}
h= & 18 v_{0}-28 w_{0} \\
& w_{1}=-55 w_{0}+36 v_{0} \\
\therefore & v_{1}=55 v_{0}-84 w_{0} \\
& u_{1}=u_{0}
\end{aligned}
$$

Hence the matrix representation of the above solution is

$$
\left[\begin{array}{l}
w_{1} \\
v_{1}
\end{array}\right]=\left[\begin{array}{ll}
-55 & 36 \\
-84 & 55
\end{array}\right]\left[\begin{array}{l}
w_{0} \\
v_{0}
\end{array}\right]
$$

where, $\mathrm{A}=\left[\begin{array}{ll}-55 & 36 \\ -84 & 55\end{array}\right]$

Repeating the above process, the general values for $v$ and $w$ are given by

$$
\begin{aligned}
& \left(\begin{array}{l}
w_{n} \\
v_{n}
\end{array}\right)=A^{n}\left(\begin{array}{l}
w_{0} \\
v_{0}
\end{array}\right) \\
& \left(\begin{array}{l}
w_{n} \\
v_{n}
\end{array}\right)=\left[\begin{array}{ll}
\frac{1^{n}}{2}(-54)+\frac{(-1)^{n}}{-2}(-56) & \frac{1^{n}}{2}(36)+\frac{(-1)^{n}}{-2}(36) \\
\frac{1^{n}}{2}(-84)+\frac{(-1)^{n}}{-2}(-84) & \frac{1^{n}}{2}(56)+\frac{(-1)^{n}}{-2}(54)
\end{array}\right]\left(\begin{array}{l}
w_{0} \\
v_{0}
\end{array}\right)
\end{aligned}
$$

Then we get the $n^{\text {th }}$ solution as

$$
\begin{aligned}
& w_{n}=\left[\frac{1^{n}}{2}(-54)+\frac{(-1)^{n}}{-2}(-56)\right] w_{0}+\left[\frac{1^{n}}{2}(36)+\frac{(-1)^{n}}{-2}(36)\right] v_{0} \\
& v_{n}=\left[\frac{1^{n}}{2}(-84)+\frac{(-1)^{n}}{2}(-84)\right] w_{0}+\left[\frac{1^{n}}{2}(56)+\frac{(-1)^{n}}{-2}(54)\right] v_{0} \\
& u_{n}=u_{0}
\end{aligned}
$$

In view of (2), the general solution of (1) is

$$
\begin{aligned}
& x_{n}=u_{n}+v_{n} \\
& =u_{0}+\left[\frac{1^{n}}{2}(-84)+\frac{1}{2}(-84)\right] w_{0}+\left[\frac{1^{n}}{2}(56)+\frac{(-1)^{n}}{-2}(54)\right] v_{0} \\
& y_{n}=u_{n}-v_{n} \\
& =u_{0}-\left[\frac{1^{n}}{2}(-84)+\frac{1}{2}(-84)\right] w_{0}+\left[\frac{1^{n}}{2}(56)+\frac{(-1)^{n}}{-2}(54)\right] v_{0} \\
& z_{n}=2 u_{n}
\end{aligned}
$$




\section{C.Pathmapriya and Dr.G.Sumathi}

$=2 u_{0}$

\section{Triple 2:}

Let $u_{0}, v_{0}, w_{0}$ be the initial solution of $(3)$

$\left.\begin{array}{l}u_{1}=u_{0}+3 h \\ v_{1}=v_{0} \\ w_{1}=w_{0}+h\end{array}\right\}$

be the second solution of (3),

Following the procedure as above, the corresponding integer solutions to (1) is given by

$$
\begin{aligned}
& w_{n}=\left[\frac{1^{n}}{2}(9)+\frac{(-1)^{n}}{-2}(7)\right] w_{0}+\left[\frac{1^{n}}{2}(-3)+\frac{(-1)^{n}}{-2}(-3)\right] u_{0} \\
& x_{n}=\left[\left[\frac{(1)^{n}}{2}(21)+\frac{(-1)^{n}}{-2}(21)\right] w_{0}+\left[\frac{(1)^{n}}{2}(-3)+\frac{(-1)^{n}}{-2}(-3)\right] u_{0}\right]+v_{0} \\
& y_{n}=\left[\left[\frac{(1)^{n}}{2}(21)+{\frac{(-1)^{n}}{-2}}^{n}(21)\right] w_{0}+\left[{\frac{(1)^{n}}{2}}^{n}(-3)+{\frac{(-1)^{n}}{-2}}^{n}(-3)\right] u_{0}\right]-v_{0} \\
& z_{n}=\left[\left[\frac{(1)^{n}}{2}(21)+{\frac{(-1)^{n}}{-2}}^{n}(21)\right] w_{0}+\left[{\frac{(1)^{n}}{2}}^{n}(-3)+{\frac{(-1)^{n}}{-2}}^{-2}(-3)\right] u_{0}\right]
\end{aligned}
$$

Triple 3:

Let $u_{0}, v_{0}, w_{0}$ be the initial solution of (3)

$$
\left.\begin{array}{c}
w_{1}=2 w_{0} \\
v_{1}=2 v_{0}+h \\
u_{1}=2 u_{0}+h
\end{array}\right\}
$$

be the second solution of $(3)$

In this case, the corresponding integer solutions to (1) is given by $x_{n}=u_{n}+v_{n}$

$=u_{0}\left[\frac{(2)^{n}}{4}(3)+\frac{(-2)^{n}}{-4}(-1)+\frac{(2)^{n}}{4}(-1)+\frac{(-2)^{n}}{-4}(-1)\right]+v_{0}\left[\frac{(2)^{n}}{4}(-3)+\frac{(-2)^{n}}{-4}(-3)+\frac{(2)^{n}}{4}(1)+\frac{(-2)^{n}}{-4}(-3)\right]$

$y_{n}=u_{n}-v_{n}$

$$
\begin{aligned}
& =u_{0}\left[\frac{(2)^{n}}{4}(3)+\frac{(-2)^{n}}{-4}(-1)+\frac{(2)^{n}}{4}(-1)+\frac{(-2)^{n}}{-4}(-1)\right]-v_{0}\left[\frac{(2)^{n}}{4}(-3)+\frac{(-2)^{n}}{-4}(-3)+\frac{(2)^{n}}{4}(1)+\frac{(-2)^{n}}{-4}(-3)\right] z_{n} \\
& =2\left[\frac{(2)^{n}}{4}(3)+\frac{(-2)^{n}}{-4}(-1)\right] u_{0}+\left[\frac{(2)^{n}}{4}(-3)+\frac{(-2)^{n}}{-4}(-3)\right] v_{0} \\
& w_{n}=2^{n} w_{0}
\end{aligned}
$$




\section{On the homogeneous cubic equation with four unknowns}

$$
\left(x^{3}+y^{3}\right)=7 z w^{2}
$$

\section{Special relations}

Employing the solutions $(x, y)$ of (1) each of following expressions among the special polygonal, centred polygonal, pronic numbers and pyramidal numbers is a congruent to zero under modulo 7.

$$
\begin{aligned}
& \text { (i) }\left(\frac{3 P_{x}^{3}}{t_{3, x+1}}\right)^{3}+\left(\frac{18 P_{x}^{3}-2}{C t_{6, x-2}-1}\right)^{3} \\
& \text { (ii) }\left(\frac{6 P_{x}^{5}}{C t_{6, x^{-1}}}\right)^{3}+\left(\frac{3 P_{y}^{3}}{t_{3, x+1}}\right)^{3} \\
& \text { (iii) }\left(\frac{P^{5} x}{t_{3, x}}\right)^{3}+\left(\frac{6 P_{y}^{3}}{C t_{6, y-1}}\right)^{3} \\
& \text { (iv) }\left(\frac{6 P_{x}^{3}}{P_{r} x}\right)^{3}+\left(\frac{P_{y}^{3}}{t_{3, y}}\right)^{3} \\
& \text { (v) }\left(\frac{2 P^{5} x}{t_{4, y}}\right)^{3}+\left(\frac{P^{5} y}{t_{3, y}}\right)^{3}
\end{aligned}
$$

\section{Conclusion}

To conclude one may search for other patterns of solutions and their corresponding properties to the considered on the homogeneous cubic equation with four unknowns.

\section{REFERENCES}

1. L.E.Dickson, History of theory of numbers and Diophantine analysis, Vol. 2, Dover publications, New York, (2005).

2. L.J.Mordell, Diophantine equations, Academic press, New York, (1970).

3. R.D.Carmicheal, The theory of numbers and Diophantine analysis, Dover publications, New York, (1959)

4. M.A.Gopalan, S.Vidhyalakshmi and G.Sumathi, On the homogeneous cubic equation with four unknowns $x^{3}+y^{3}=14 z^{3}-3 w^{2}(x+y)$, Discovery science, 2(4) (2012) 1719.

5. M.A.Gopalan, S.Vidhyalakshmi and G.Sumathi, On the homogeneous cubic equation with four unknowns $x^{3}+y^{3}=z^{3}+w^{2}(x+y)$, Diophantine J. Math, 2(2) (2013) 99103.

6. On the ternary non-homogeneous cubic equation $x^{3}+y^{3}+z\left(x^{2}+y^{2}-20\right)=4(x+y)^{2} z$, Impact J. Sci, Tech, 7(2) (2013) 1-6.

7. on the homogeneous cubic equation with three unknowns $x^{3}+y^{3}=14 z^{3}+3(x+y)$, Discovery Science, 2(4) (2012) 37-39. 